\title{
In-cloud formation of secondary species in iron-containing particles
}

Qinhao Lin ${ }^{1}$, Xinhui Bi ${ }^{1}$, Guohua Zhang ${ }^{1}$, Yuxiang Yang ${ }^{1,2}$, Long Peng ${ }^{1,2}$, Xiufeng Lian ${ }^{1,2}$, Yuzhen $\mathrm{Fu}^{1,2}, \mathrm{Mei}^{3}$, Duohong Chen ${ }^{4}$, Mark Miller ${ }^{5}$, Ji Ou${ }^{6}$, Mingjin Tang ${ }^{1}$, Xinming Wang ${ }^{1}$, Ping'an Peng ${ }^{1}$, Guoying Sheng ${ }^{1}$, and Zhen Zhou $^{3}$

${ }^{1}$ State Key Laboratory of Organic Geochemistry and Guangdong Key Laboratory of Environmental Resources Utilization and Protection, Guangzhou Institute of Geochemistry, Chinese Academy of Sciences, Guangzhou 510640, PR China

${ }^{2}$ University of Chinese Academy of Sciences, Beijing 100039, PR China

${ }^{3}$ Institute of Mass Spectrometer and Atmospheric Environment, Jinan University, Guangzhou 510632, PR China

${ }^{4}$ State Environmental Protection Key Laboratory of Regional Air Quality Monitoring, Guangdong Environmental Monitoring Center, Guangzhou 510308, PR China

${ }^{5}$ Department of Environmental Sciences, Rutgers, the State University of New Jersey, New Brunswick, NJ 08901, USA

${ }^{6}$ Shaoguan Environmental Monitoring Center, Shaoguan 512026, PR China

*Correspondence to: Xinhui Bi (bixh@gig.ac.cn) 


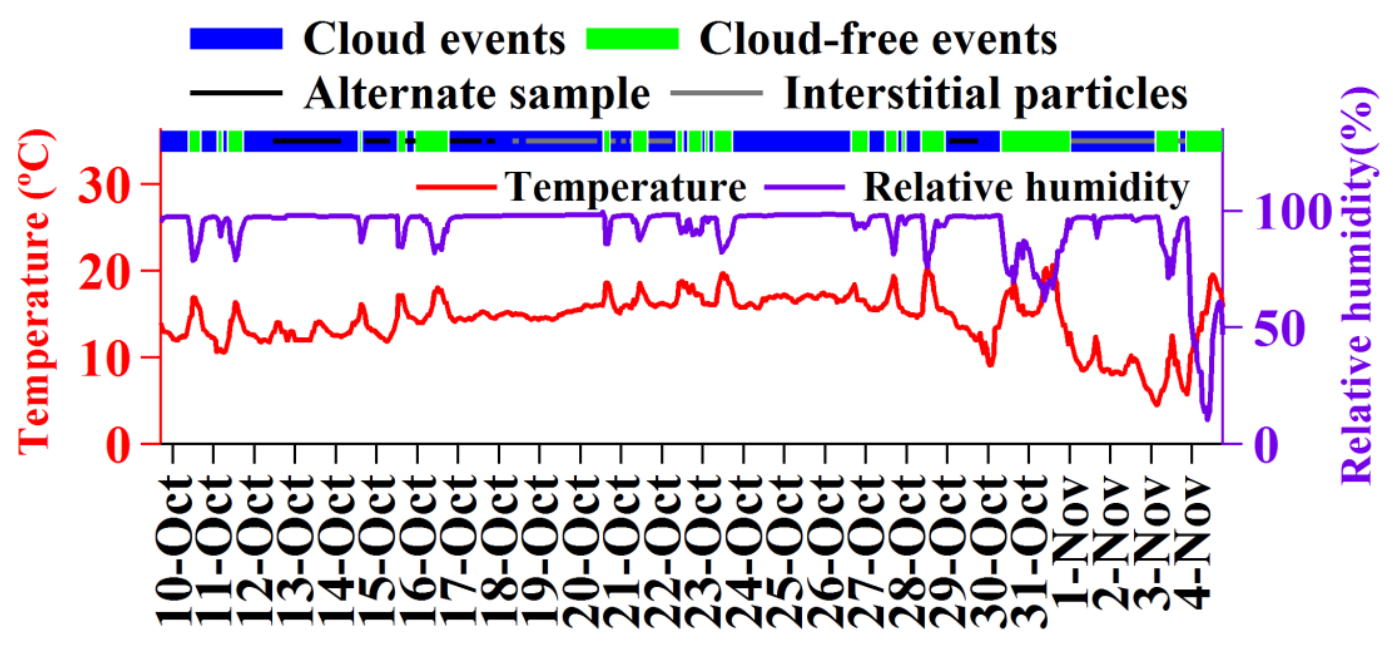

Figure S1. Hourly mean in temperature and relative humidity during the study period. The black lines represent the period that GCVI and $\mathrm{PM}_{2.5}$ inlets alternately sample with an interval of one hour. The $\mathrm{PM}_{2.5}$ inlet (gray

29 lines) was only used to correct interstitial particles during cloud processing. 


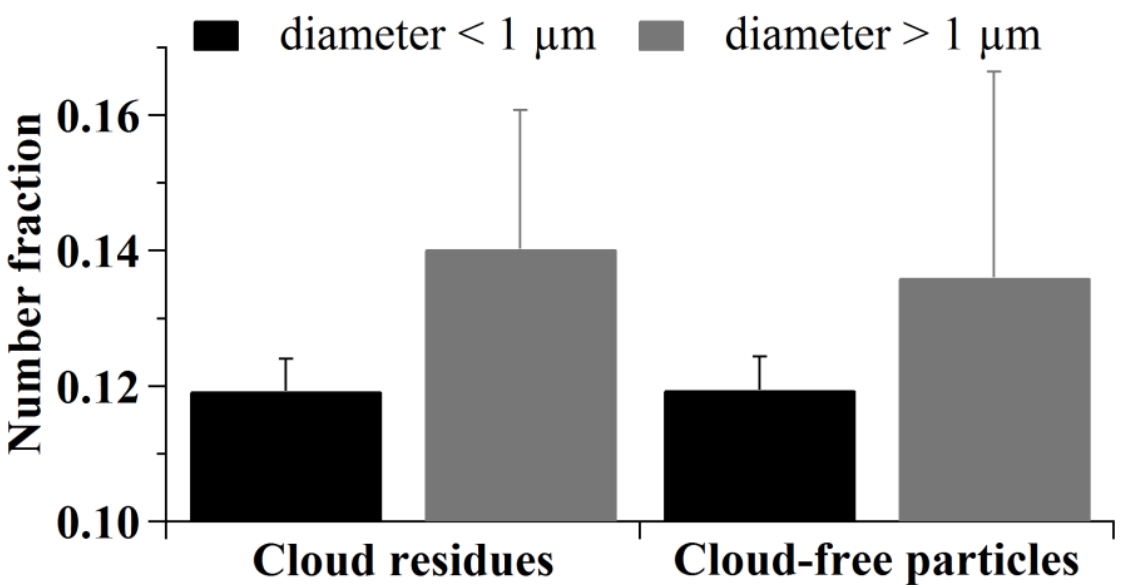

32

33 Figure $\mathrm{S} 2$. The number fraction of the Fe-dust cloud residues and cloud-

34 free particles in diameter $<1 \mu \mathrm{m}$ and $>1 \mu \mathrm{m}$. 


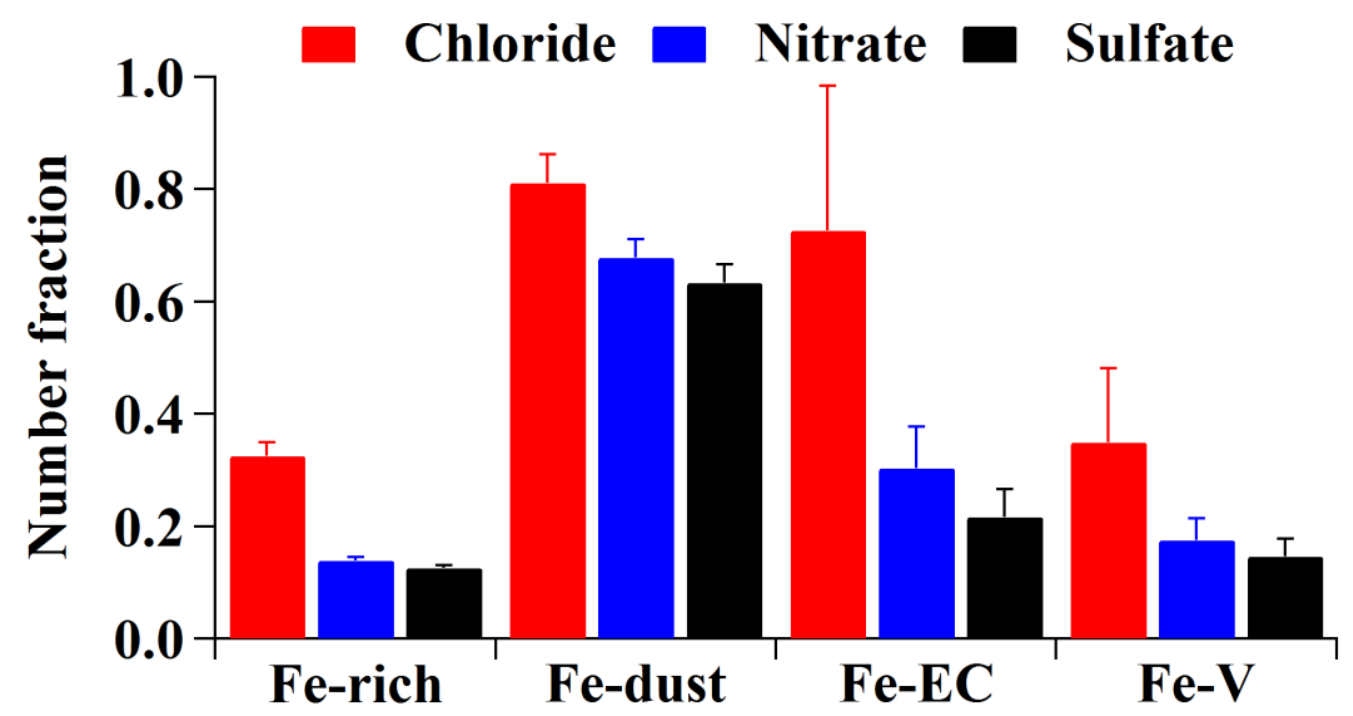

35

36 Figure S3. Number fractions of chloride, nitrate, sulfate, and oxalate

37 internally mixed with the Ca-containing particles in the four Fe-containing 38 cloud residual types. 


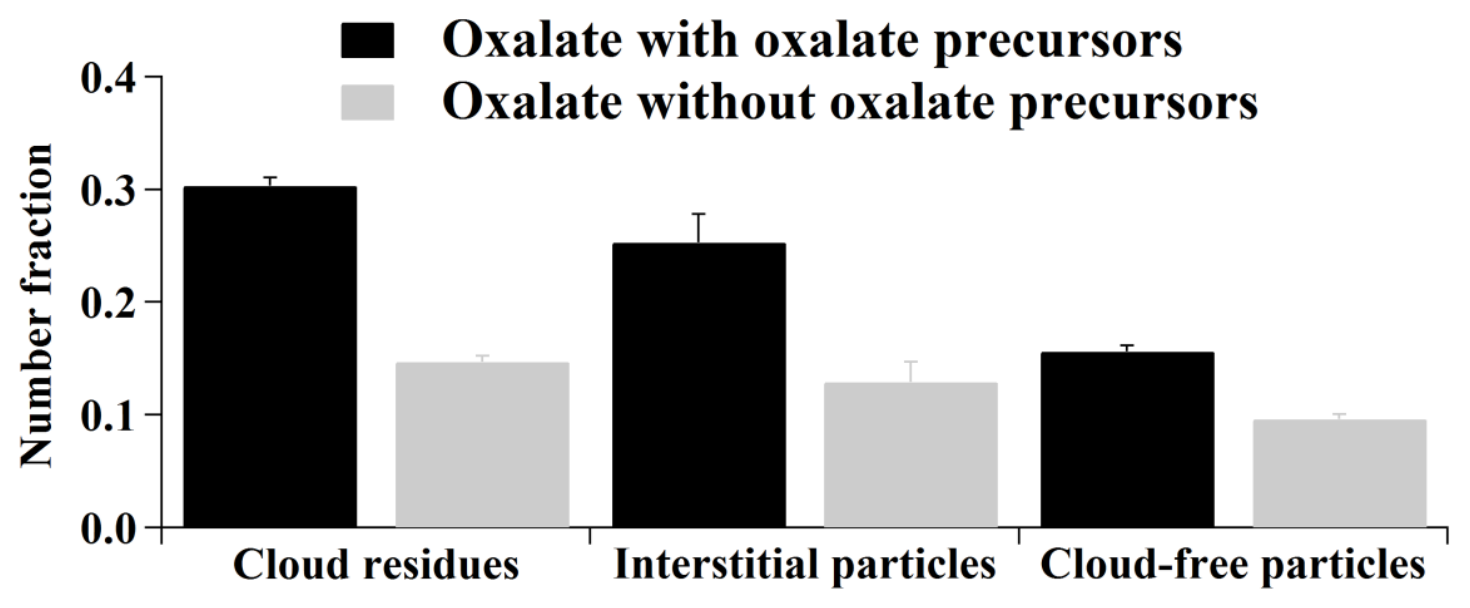

40 Figure S4. Number fraction of oxalate with and without its precursor in the

41 Fe-containing cloud residues, interstitial particles, and cloud-free particles. 


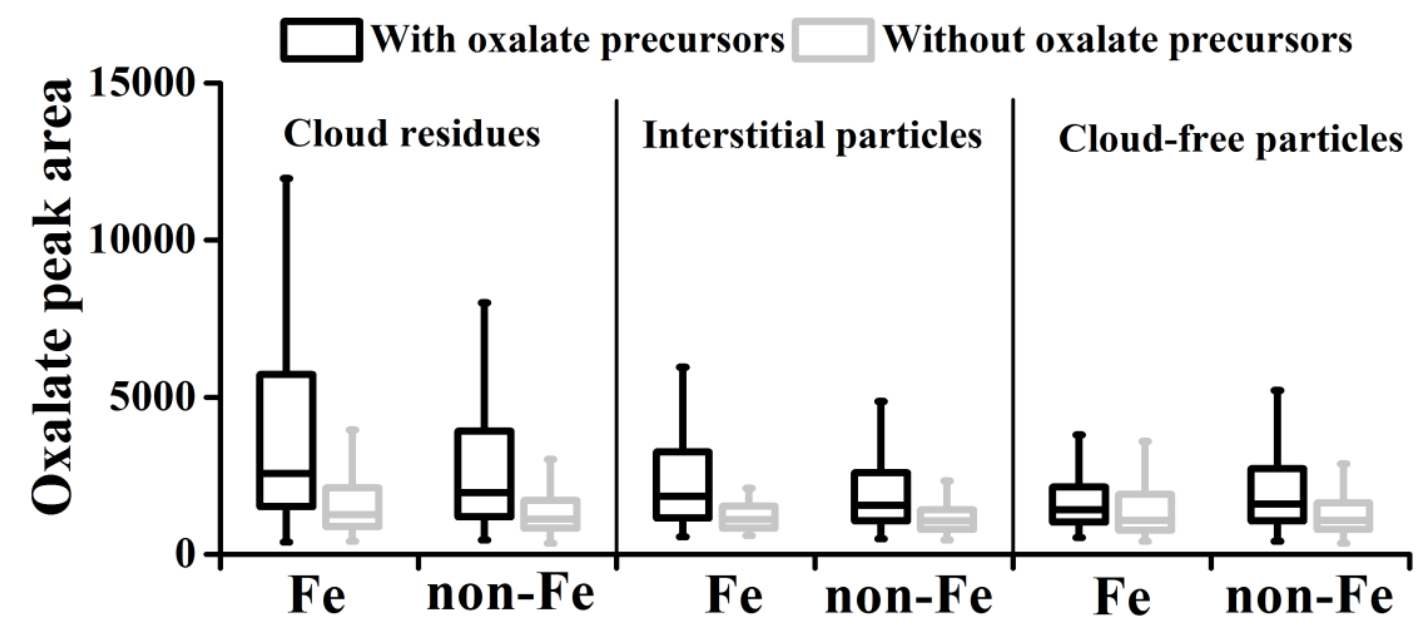

44

45 Figure S5. The comparison for oxalate peak area in with and without oxalate precursors. 


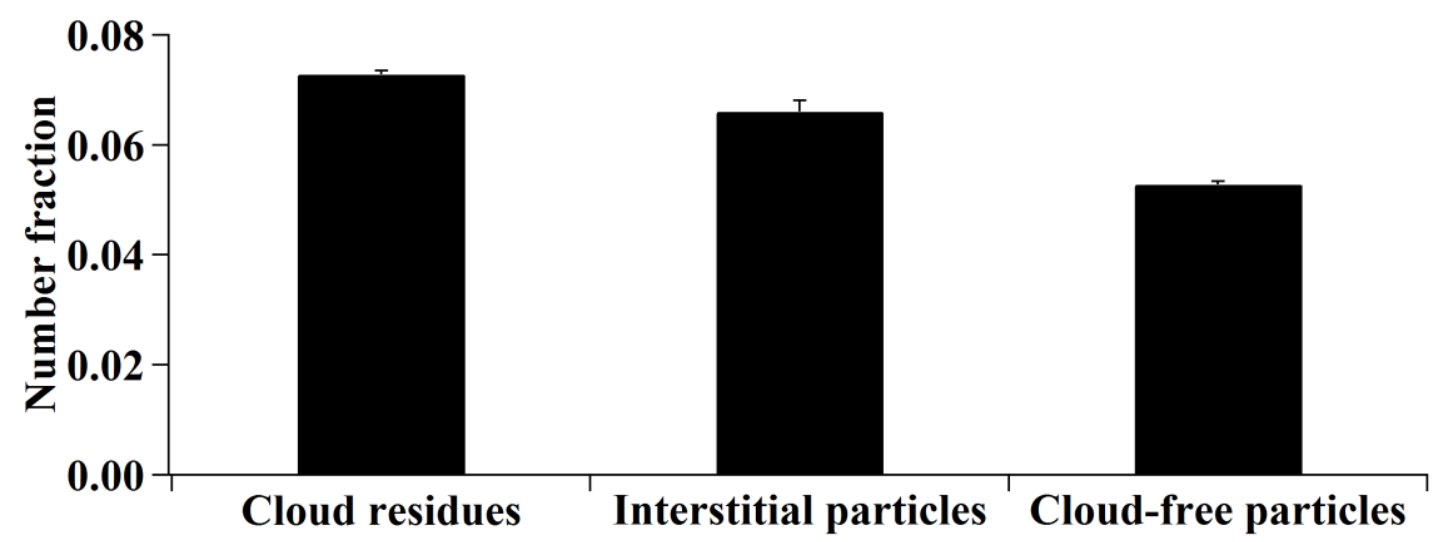

48

49 Figure S6. Number fraction of oxalate in the non-Fe cloud residues, 50 interstitial particles, and cloud-free particles. 

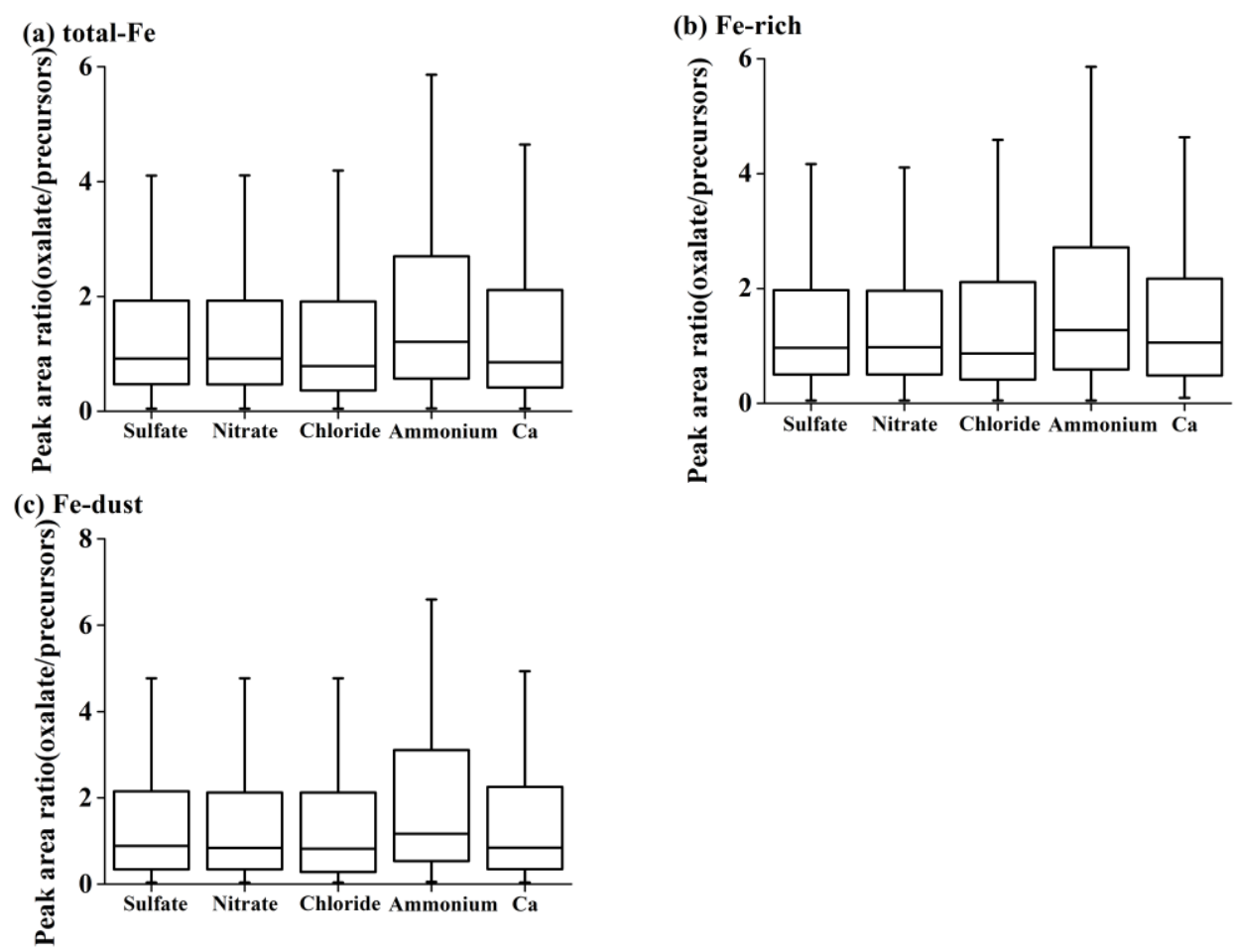

52 Figure S7. Peak area ratio of oxalate to its precursors with sulfate, nitrate,

53 chloride, ammonium, and $\mathrm{Ca}$ in the $\mathrm{Fe}$-containing cloud residues. 